\title{
High Resolution 3D Reconstructions of Rocks and Composites
}

\author{
E. Rosenberg ${ }^{1}$, J. Lynch ${ }^{1}$, P. Guéroult ${ }^{1}$, M. Bisiaux ${ }^{1}$ and R. Ferreira De Paiva ${ }^{1}$ \\ 1 Institut français du pétrole, 1 et 4, avenue de Bois-Préau, 92852 Rueil-Malmaison Cedex - France \\ e-mail: elisabeth.rosenberg@ifp.fr
}

\begin{abstract}
Résumé - Reconstructions 3D haute résolution de roches et de composites - Des représentations tridimensionnelles de différents milieux ont été obtenues avec une résolution de $10 \mu \mathrm{m}$ en utilisant un microscanner développé au laboratoire à partir d'une microsonde électronique. Peu de modifications ont été nécessaires. Certains éléments de la microsonde ont même été conservés pour assurer la stabilité de l'acquisition et permettre l'obtention de radiographies haute résolution $(2 \mu \mathrm{m})$. L'impact d'un faisceau d'électrons focalisé sur un film mince constitue la source $\mathrm{X}$ et l'image radiographiée de l'échantillon est acquise à l'aide d'une caméra CCD. Un mécanisme de rotation de l'échantillon permet l'acquisition de radiographies à différentes positions angulaires ainsi que la reconstruction de la cartographie 3D des coefficients d'atténuation. L'atténuation des rayons $\mathrm{X}$ étant directement reliée à la densité et au numéro atomique du matériau irradié, le microscanner fournit une cartographie 3D des différentes phases présentes dans l'échantillon. Les performances du système ont été testées sur divers échantillons, principalement des roches et des matériaux composites. La comparaison avec des images de microscopie électronique à balayage a été utilisée pour valider les reconstructions. Les résultats sont pour la plupart qualitatifs mais montrent déjà le potentiel de cette technique pour décrire la topologie et la connectivité 3D de milieux poreux ou l'orientation des fibres dans des composites renforcés de fibres de verre.

Mots-clés : microscanner, texture, roches, fibres, matérieux composites, 3D, porosité.
\end{abstract}

\begin{abstract}
High Resolution 3D Reconstructions of Rocks and Composites - Ten $\mu$ m resolution 3D representations of different media, were obtained with a laboratory computer microtomograph developed from an electron microprobe column. From the original electron microprobe, only minor modifications have been required, indeed several of the utilities of the microprobe have been used to ensure high resolution radiography $(2 \mu \mathrm{m})$. The impact of the electron beam focused onto a thin film is used to form a "point" X-ray source and the radiographic image of the sample is acquired on a CCD camera. A specimen rotation mechanism allows multiple radiograph acquisition and reconstruction of the X-ray attenuation $3 D$ cartography. Since X-ray attenuation is directly related to density and atomic number, the microscanner provides $3 D$ cartographs of the different phases present in the sample. System performances have been evaluated on various samples, mainly rocks and composites. Comparison with scanning electron micrographs was used when possible to validate the reconstructions. Results are mostly qualitative but already show the potential of the technique in describing $3 D$ connectivity and topology of pore networks or $3 D$ orientation of fibres in composites.
\end{abstract}

Keywords: microtomography, texture, rocks, fibres, composites, 3D, porosity. 


\section{INTRODUCTION}

With the use of synchrotron radiation as a photon source, $\mathrm{X}$-ray microtomography has emerged as a technique that can be applied in material science for obtaining 3D images with a spatial resolution in the 1-20 $\mu \mathrm{m}$ range (Coles, 1994-1996). Due to the difficulty of access of synchrotron sources, there have been many attempts to obtain a similar performance by use of laboratory equipment (Cazaux 1989; Cheng, 1992). This has been made possible by recent developments in the field of detectors and computers towards high spatial resolutions and fast data acquisition. One aspect of our work concerns the development of such an equipment, the second being to show the potential of this technique and its complementarity with the well known scanning electron microscopy in describing materials at the micron scale.

\section{METHODS}

The microscanner uses an electron microprobe as a "point" X-ray source (Ferreira De Paiva, 1996). This system, based on the original idea of Sasov (Sasov, 1987) and following the development of Cazaux et al. (Cazaux, 1989), is capable of $2 \mu \mathrm{m}$ resolution radiographic imaging. The basic principle is to use the interaction volume of the focused electron beam with a thin target as an X-ray source. The target is fixed on the original sample holder. The object is fixed on a horizontal rotation axis, built in house, placed between this source and a phosphor screen. The projected image of the object (radiography) is acquired for each angular position of the object. Magnification is changed by varying the position of the target. The projected X-ray micrograph is converted to an equivalent light image by the phosphor screen. The light image is demagnified by a factor of 1.6 onto a CCD camera via a camera objective lens working in macro configuration. The CCD used is a digital pixel large format (maximum $1152 \infty 770$ pixels) Peltier cooled $\left(-20^{\circ} \mathrm{C}\right)$ device with a pixel size of $22.5 \mu \mathrm{m}$ and a dynamic range of 4096 . The apparatus is presented in schematic form in Figure 1.

The equivalent of the CCD pixel size on the phosphor screen is thus $22.5 \infty 1.6=36 \mu \mathrm{m}$. Resolution below this depends on the intrinsic magnification of the radiograph. Magnifications of over 100 are achievable, reducing the equivalent detector element size to $0.36 \mu \mathrm{m}$. Such detector resolutions are seldom useful due to spatial resolution limitations induced by the electron beam size. However acquisition time can be greatly reduced by pixel binning. In the results presented here, $3 \infty 3$ binning was used to obtain $256 \infty 256$ images using $66 \%$ of the active surface of the CCD whilst retaining the possibility of a one $\mu \mathrm{m}$ resolution limit of the detection system. Typically the acquisition time is of the order of one minute per radiograph.

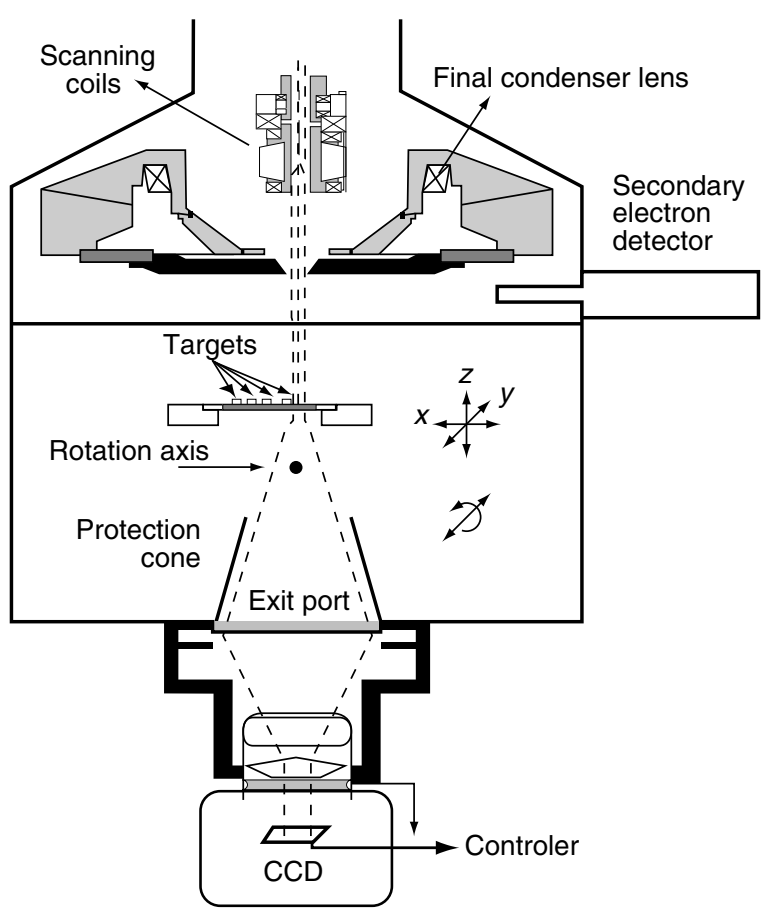

Figure 1

Schematic representation of the microtomograph.

The two dimensional nature of the CCD detector means that microradiographs are produced directly without the need for beam or specimen displacement. For each specimen, a series of 120 (or 240) images ( $256 \infty 256$ pixels) is recorded by rotating the specimen by $3^{\circ}$ (or $1.5^{\circ}$ ) between successive radiographs. A laps of time is observed between a rotation and the corresponding acquisition to enable the fading of residual remanence.

Raw data are then treated in order to convert them to log I/I0 data. The reconstruction software, developed from that of Sasov (Sasov, 1987), uses the filtered back projection method and assumes parallel beam geometry which is a good approximation as long as the opening angle is less than $10^{\circ}$ which is the case in our configuration.

The reconstruction time on a PC computer is of the order of one minute per section ( $256 \propto 256$ pixels). The result is a $256 \infty 256 \infty 256$ three dimensional microtomograph of the sample. Although in principle 400 angular steps are required to obtain a good reconstruction, it was found that 120 steps did not produce significant reconstruction artefacts whilst limiting the total acquisition time.

It must be noted that the reconstruction size is currently limited to $(256)^{3}$ voxels so that specimen size is limited to submillimetric sizes if a high resolution (better than $10 \mu \mathrm{m}$ ) is needed. Visualisation of volumic data is made via the Spyglass slicer software on PC. The whole analytical procedure is sketched on Figure 2. 


\section{ROSEMB02}

Figure 2

Schematic representation of the analytical procedure.

\section{RESULTS}

\section{1 Rocks}

The three dimensional pore structure of reservoir rocks and the wetting of porous media by fluids are of great importance in oil recovery. The transport properties of porous media are determined by their microstructure, i.e. geometry and topology of interconnected pore space. Extraction of petroleum by water injection depends on the displacement of oil in place by water and is also known to be strongly influenced by the wettability of reservoir minerals by water relative to oil (Cuiec, 1991). On the macroscopic scale several groups, among whom IFP, have the applied three dimensional imaging possibilities offered by X-ray scanner technology to the study of fluid interactions with reservoir rocks. Tomography using medical scanners does not however reach the spatial resolution necessary for direct visualisation of the pore structure or of the fluids in place. On the other hand, scanning electron microscopy leads to a detailed description of the core at the sub-micron scale but remains a 2D characterisation and gives incomplete information on the topology of the pore network. This laboratory microscanner enables the reconciliation of both techniques by providing 3D images with a resolution of the order of $10 \mu \mathrm{m}$ in simple conditions and with reasonable delays .

To study the reservoir rocks, we need a high average $\mathrm{X}$-ray energy. This was achieved using a niobium target with a characteristic line at $16.6 \mathrm{keV}$ supported on carbon, excited by a $30 \mathrm{keV}$ primary electron beam energy.

\subsubsection{Model Porous Media}

Model porous media were created by filling $1 \mathrm{~mm}$ diameter borosilicate glass capillary tubes with silica glass beads of 30 to $200 \mu \mathrm{m}$ diameter. A negative of a radiographic projection and a tomographic reconstruction of the model porous medium are shown in Figure 3 (a, b, c). On the radiographic projection (Fig. 3a) each pixel of the image is an integral measurement of the attenuation coefficient along the line connecting the source to each cell of the detector. Structures appear as if they were placed on top of each other. A 3D representation of the capillary tube with a wedge removed is shown in Figure 3b. The morphology of the glass beads is clearly reproduced. On this reconstruction, we can locate each detail of the structure.

An SEM image of a polished section of the sample is shown in Figure 3d. The 3D visualisation software enables cropping the reconstructed object and extraction of a chosen volume of the sample (Fig. 3c). In this case, bubble type faults, $20 \mu \mathrm{m}$ in diameter, clearly visible on both SEM images (Fig. 3d) and the 3D reconstruction (Fig. 3c), attest to the resolution performance of the instrument.

The images in Figure 4 show that 3D fluid distribution can be studied in this kind of medium provided that fluids are marked with a heavy element. In the case presented here, water was marked with high concentration $(300 \mathrm{~g} / 1)$ potassium bromide and added to a water wet and then an oil wet medium.

The radiographic images (Fig. 4a, 4d and 4e) and reconstructed sections (Fig. $4 \mathrm{~b}$ and $4 \mathrm{c}$ ) or volume (Fig. 4f) 

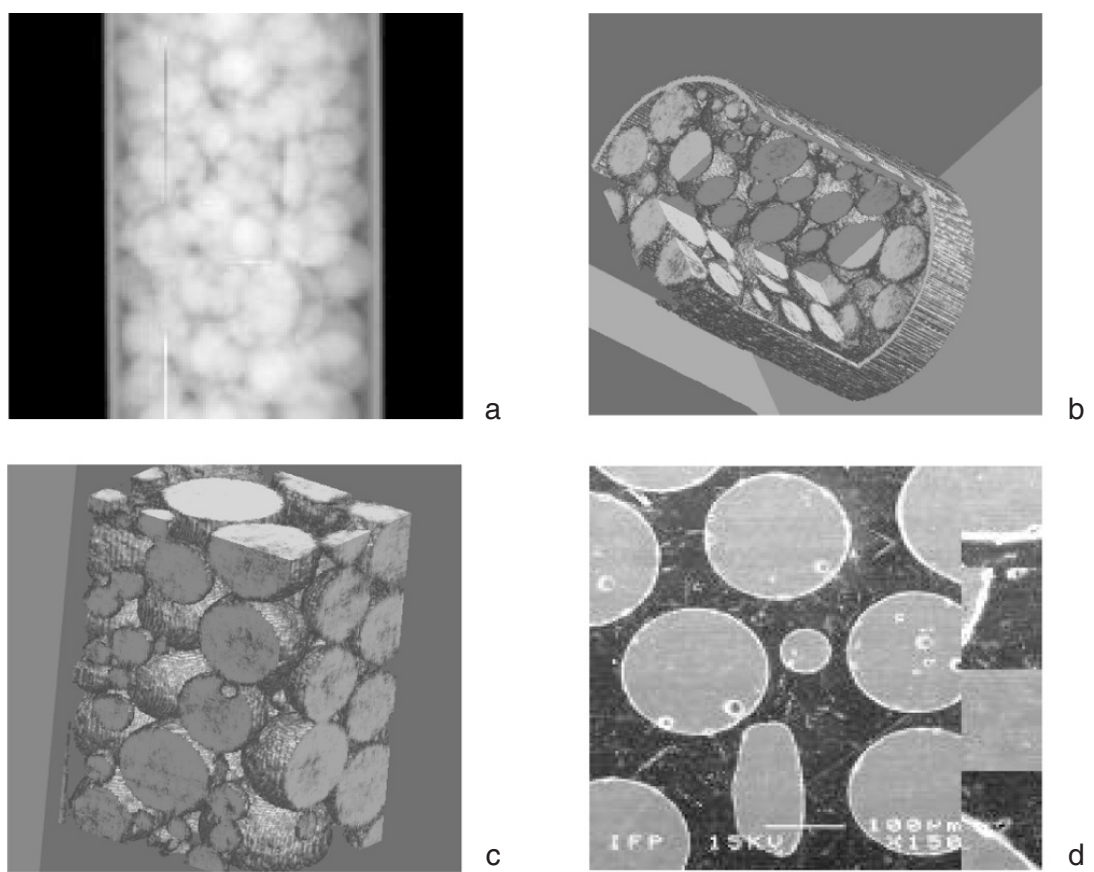

Figure 3

Model porous medium: glass beads in a capillary tube. Radiographic image (a), reconstructed volume (b), part of the reconstructed volume (c) and SEM image the beads (d).
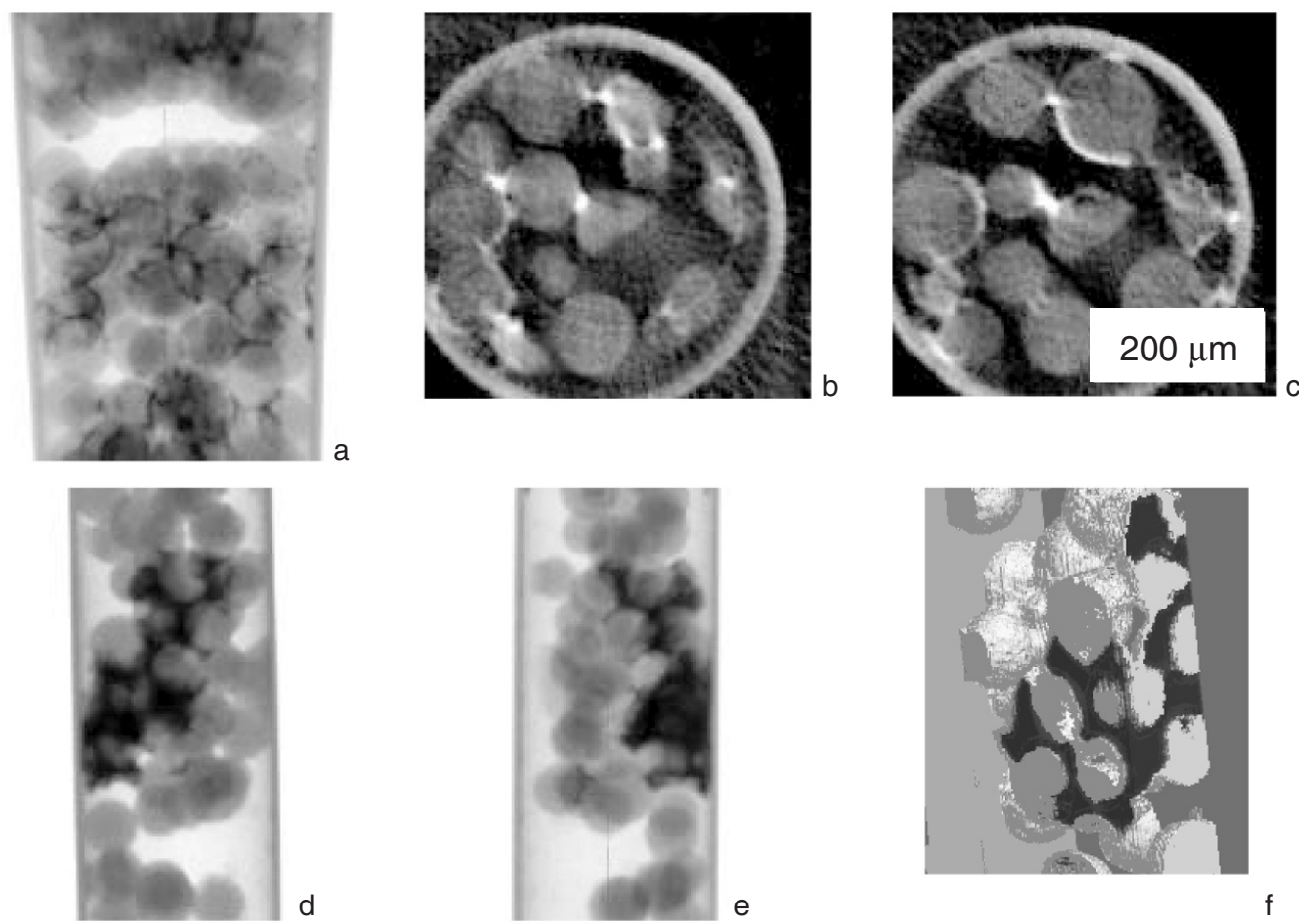

Figure 4

Water distribution in different wettability media. a, b, c: water wet medium. Radiographic image (a) and reconstructed sections (b and c). Water is represented in white. d, e, f: oil wet medium. Radiographic image (d and e) and reconstructed volume (f). Water is represented in black. 
show clearly the different distributions of water depending on the wettability of the medium:

- water appears in the form of thin films and menisci in the water wet medium;

- water appears in the form of a large 3D globule in the case of the oil wet medium.

The main drawbacks in this application are the size of the sample (maximum width $3 \mathrm{~mm}$ ) which make boundary effects not negligible, and the acquisition time, still too long to enable dynamic displacement of fluids within the sample to be studied. Further investigations have focused on the description of the framework and pore network of rocks.

\subsubsection{Real Rocks}

Real rocks have been prepared from raw or resin embedded fragments of rocks, 1 to $2 \mathrm{~mm}$ in diameter and 2 to $4 \mathrm{~mm}$ in length. A model rock of Fontainebleau sandstone and a carbonate sample are first used to show the reliability of the reconstructions. For this, the external surfaces of the reconstructed volumes are compared to equivalent SEM images. Then, a set of samples from a sandstone reservoir in the Brent formation of the North Sea field (oil zone) is presented and 3D measurements are compared to global and mercury intrusion analyses.

\section{Clean Sandstone and Carbonate}

Fontainebleau sandstone is a clay free sandstone often used as a model reservoir rock. A small piece of rock has been glued to the sample holder. 256 consecutive sections have been reconstructed from projections ( $6 \mu \mathrm{m}$ pixel size). Figures $5 \mathrm{a}, 5 \mathrm{~b}, 5 \mathrm{c}$ show the outer surfaces of the reconstructed tomograph to be compared with SEM images of the same sample (Fig. 5d, 5e, 5f). Although the SEM images are of higher resolution, individual grains can clearly be seen in the tomographs. These images show the reliability of the reconstruction. Note that the epoxy glue used to fix the sample onto the rotation axis, clearly visible on the SEM images, is transparent to the X-rays used and does not hinder the visualisation of sub-surface grains.

In order to study the porosity the reconstructed volume can be cropped (Fig. 5h). The reconstructed sections are
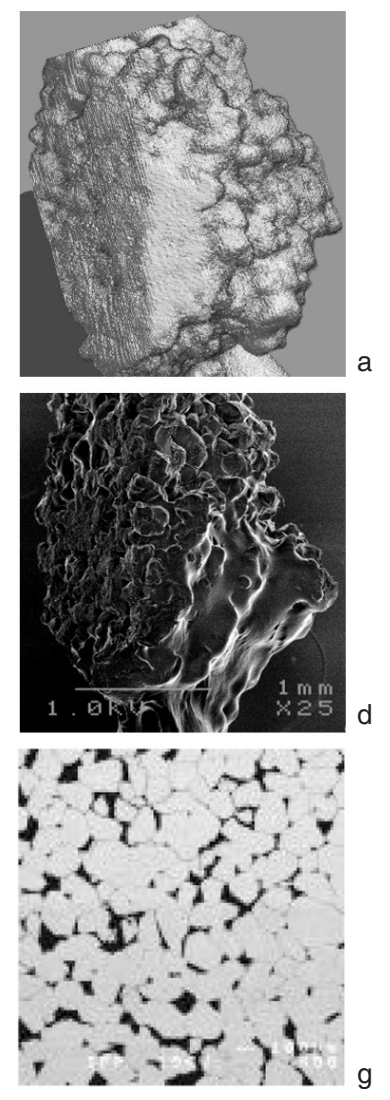
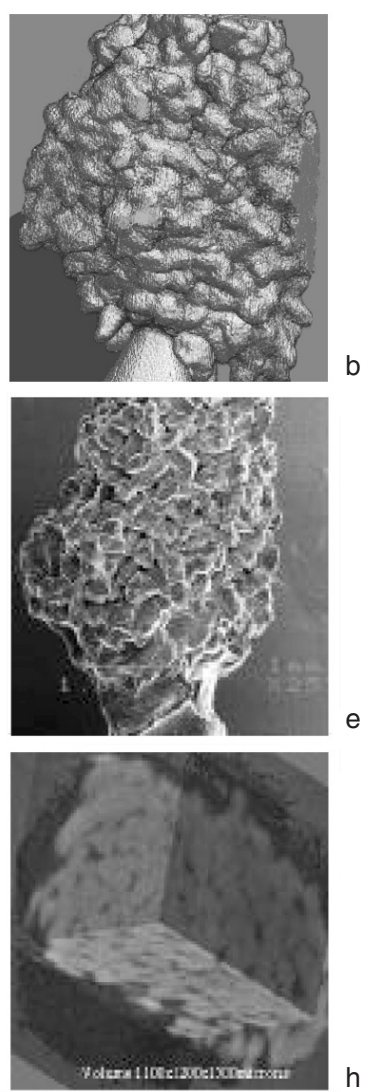
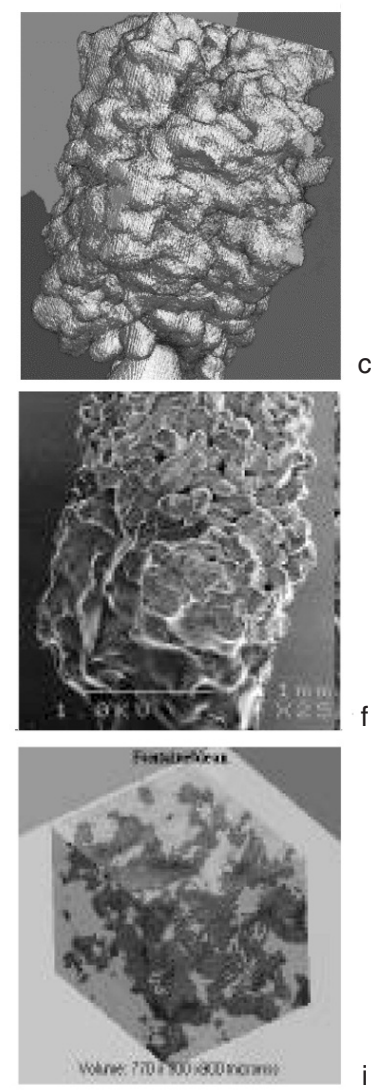

Figure 5

Fontainebleau sandstone. a, b, c: reconstructed external surfaces of the sample; d, e, f: corresponding SEM images (secondary electron images); g: SEM backscattered electron image of a rock section; $h$ and i: microscanner reconstruction of cross sections (h) and pore network (i). 
comparable to backscattered electron SEM images made on polished samples (Fig. 5g). The advantage of the microtomograph is to allow the description of $3 \mathrm{D}$ topology of the pore network and interconnectivity of pores (Fig. 5i). In this case it is clear that pores are connected from one end of the sample to the other via a network of large pore throats. This network ensures easy flow of fluids through the rock.

The same comparison can be made on a sample of a limestone (Saint-Maximin limestone) although the matrix is more absorbant than quartz. We can notice many common textural details, detectable both on SEM images (Fig. 6d, 6e, 6f) and microtomography reconstructions (Fig. 6a, 6b, 6c).

\section{Reservoir Rocks}

The following step in the study of rocks is to characterise real reservoir rocks with a large mineralogical composition and known petrophysical characteristics. Performances of the microscanner are illustrated on three samples from the Brent formation of the Alwyn North Sea field. These samples are characterised by permeabilities varying from 50 to $560 \mathrm{mD}$ and exhibit different behaviours in terms of wettability.

Their mineralogical composition is deduced from X-ray diffraction, X-ray fluorescence and electron microprobe analyses (Table 1).
TABLE 1

Global analysis of the reservoir rock samples (from Durand, 1996)

\begin{tabular}{l|l|l|l|l}
\hline & Units & CAR1 & CAR3 & CAR4 \\
\hline Porosity & $\% \mathrm{vol}$ & 15.9 & 16.6 & 22.6 \\
\hline Permeability & $\mathrm{mD}$ & 50 & 340 & 560 \\
\hline Kaolinite & $\% \mathrm{w} / \mathrm{w}$ & 0 & 3.1 & 7.8 \\
\hline Illite & $\% \mathrm{w} / \mathrm{w}$ & 13.8 & $<1$ & 0.8 \\
\hline Quartz & $\% \mathrm{w} / \mathrm{w}$ & 83.7 & 92.5 & 67.4 \\
\hline K-feldspars & $\% \mathrm{w} / \mathrm{w}$ & 0 & 3.3 & 16.8 \\
\hline Wettability & & pref. oil wet & pref. water wet & pref. oil wet \\
\hline
\end{tabular}

Mineral Framework

Sample CAR4 contains both kaolinite and feldspars and has been used to test the capabilities of the microscanner in terms of identification of minerals.

Kaolinite is an important mineral in reservoir rocks because of its affinity with oil. This mineral is difficult to identify and locate on reconstructed images because its absorption coefficient is very close to that of quartz and the size of kaolinite aggregates varies generally from 10 to $20 \mu \mathrm{m}$, i.e. close to the resolution limit of the microtomograph.
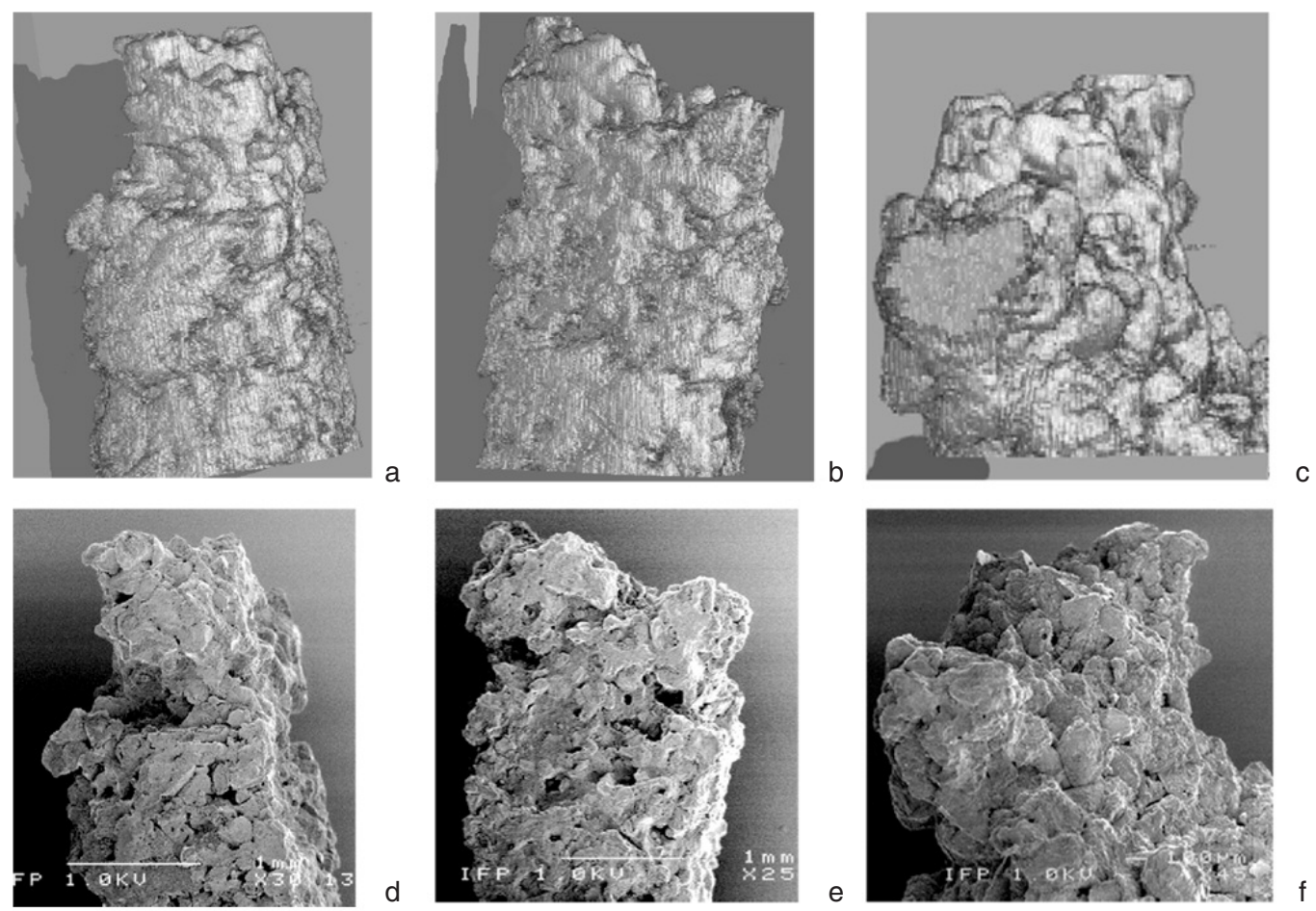

Figure 6

Saint-Maximin limestone. a, b, c: reconstructed surfaces of the sample; d, e, f: corresponding SEM images (secondary electron images). 
Detecting them will depend on the way they are aggregated. As illustrated by the following three SEM images (Fig. 7a, 7b, 7c) they can be dispersed platelets, loose aggregates, or compact aggregates (see Figures $7 \mathrm{~d}, 7 \mathrm{e}, 7 \mathrm{f}$ ).

Figure $7 \mathrm{~g}$ is a reconstruction of a small specimen of sample CAR4. This sample was less than $1 \mathrm{~mm}$ in diameter in order to reach a resolution better than $10 \mu \mathrm{m}$ on tomographic reconstructions. An SEM image of the same area is presented Figure $7 \mathrm{~h}$. In the first boxed area (Fig. 7a), kaolinite is aggregated into packs $15 \mu \mathrm{m}$ in diameter about $15 \mu \mathrm{m}$ apart . On the microscanner reconstruction (Fig. 7g), this zone is correctly reproduced with a detail enabling the identification of kaolinite on reconstructions from its fine texture. On the boxed areas (b) and (c), kaolinite packs have the same size but their dispersion is less regular (Fig. $7 \mathrm{~b}$ and 7c). Tightly assembled kaolinite (Fig. 7c) appears on the reconstruction as a massive phase with a texture and a grey level very similar to quartz, while loose aggregates of kaolinite (Fig. 7b) appear with a grey level very near to porosity. From this comparison, we can appreciate that the resolution of the reconstruction is critical to distinguish kaolinite in all cases. The limits on identification, location and quantification of clays are clear from these examples. Improving the spatial resolution will only push the problem to shorter length scales.

Feldspars on the other hand, are much more absorbent than quartz and appear on reconstructions with a high contrast. Moreover they are generally compact grains a few hundred micron diameter, much larger than the resolution limit. For these reasons, 3D distribution of feldspars in the rock can be easily described by segmentation of 3D data as illustrated on Figure 8.
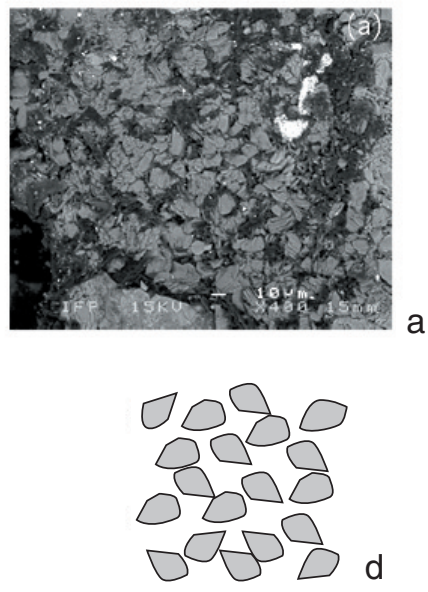

Dispersed platelets

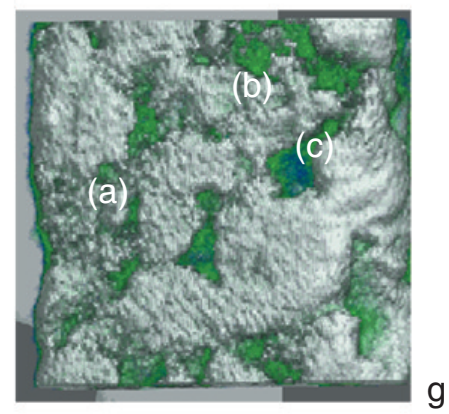

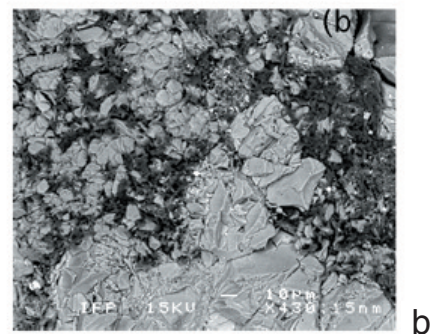

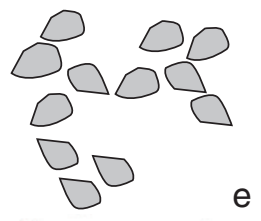

Loose aggregates

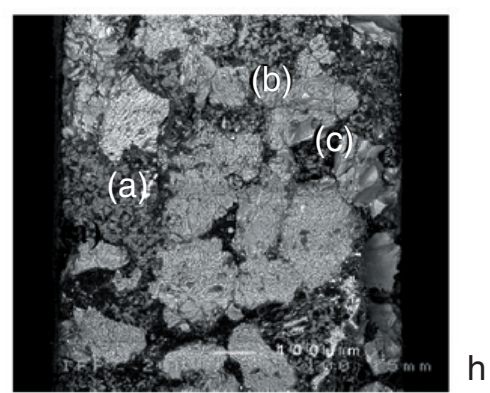

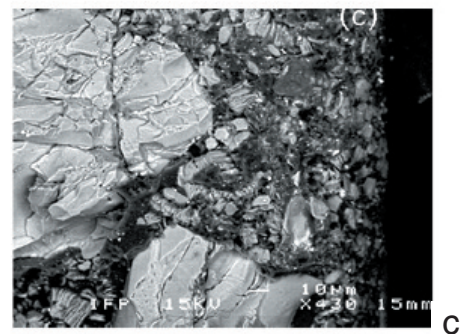

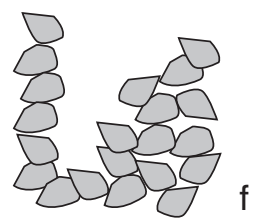

Compact aggregates

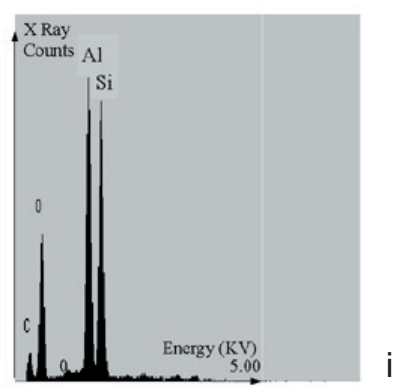

Figure 7

Kaolinite distribution within CAR4 sample. a, b, c: SEM magnification of kaolinite rich areas; d, e, f: shematic representation of the state of aggregation of kaolinite; g: microscanner reconstruction of the sample (boxes indicate the location of areas a, b, c); h: SEM image of the same area (boxes indicate the location of areas a, b, c); i: SEM/EDS spectrum of area (a) confirming kaolinite identification $\left(2 \mathrm{SiO}_{2}, \mathrm{Al}_{2} \mathrm{O}_{3}, 2 \mathrm{H}_{2} \mathrm{O}\right)$. 


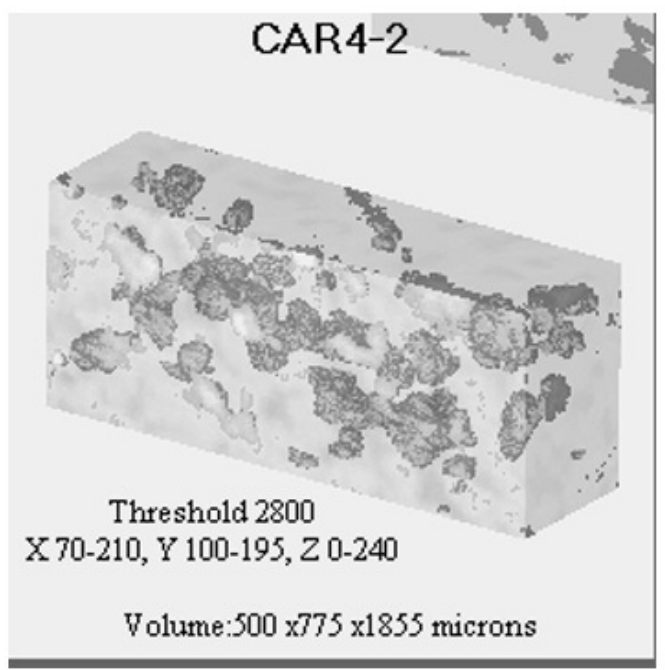

Figure 8

Feldspar grain distribution within CAR4 sample.
Pore Networks

To enable the comparison of the pore networks of the samples, larger samples were studied (up to $2 \mathrm{~mm}$ in diameter, $3 \mathrm{~mm}$ length). Each sample was described by a set of 250 slices ( $256 \infty 256$ pixels). Pixel size depends on the sample size and is respectively 8,7 and $5.5 \mu \mathrm{m}$ in the $x, y$ directions, and $11.5,10.5$, and 7.5 in the $z$ direction for CAR1, CAR3 and CAR4 samples.

Figure 9 enables comparison between backscattered electron SEM images of polished sections (Fig. 9a, 9b, 9c), and representations of the reconstructed volumes of the three samples (Fig. 9d, 9e, 9f). The volume size and voxel size are indicated below each representation. The information given in both representations is comparable: size and form of pores, contrast between quartz and feldspars grains, contrast between quartz grains and porosity.

From these images it is very difficult to characterise the connectivity of pores because apparently distinct objects in a $2 \mathrm{D}$ image can be cross sections of interconnected void space or isolated pores. Correlation with permeability is thus difficult from these $2 \mathrm{D}$ representations.
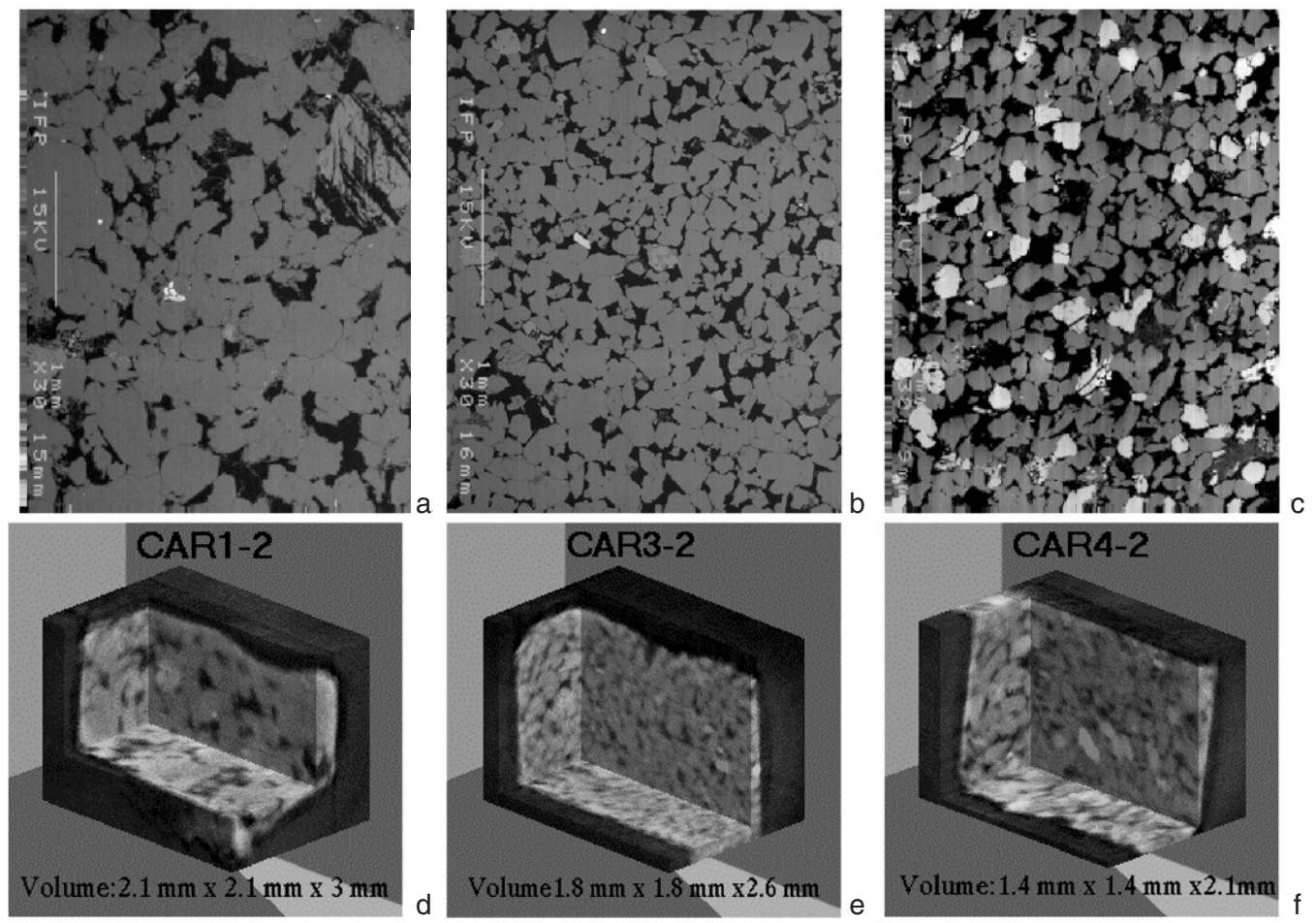

Figure 9

Comparison of SEM images and reconstructed sections of real rock samples. a, b, c: SEM backscattered electron images on polished sections of three samples of reservoir rocks (respectively, CAR1, CAR3 and CAR4); d, e, f: microtomograph reconstructions of the same samples. 
Porosity Measurements

Isolating the $3 \mathrm{D}$ pore network implies the choice of segmentation thresholds. This choice is based on the analysis of the grey level histogram. For each sample grey level histograms have been calculated from a set of 200 sections entirely included in the rock. Since they do not show well separated contributions, peaks had to be deconvoluted. From this deconvolution, we can get a semi-quantitative assessment of porosity and volume fraction of each phase. Peaks were assumed to be gaussian and a fit was realised by minimising square differences between model and data. All the histograms can be described as a combination of three peaks, four in the case of sample CAR4 because of the presence of feldspars (Fig. 10). The first corresponds to the pores with a diameter over the resolution limit. The second peak has two contributions: pores under the resolution limit and loose clay aggregates. The main peak is attributed to quartz.

The \% area of each peak has been measured and compared with global measurements from Table 1. Results are given in Table 2. Global measurements give the total pore volume fraction while measurements from histograms give separately the volume fraction of macropores $(>15 \mu \mathrm{m})$ and the volume fraction of micropores $(<15 \mu \mathrm{m})$ plus clays. Comparison of the total volume fraction of pores plus clays shows good agreement between tomographic data and global analyses from Table 1 .

Let us now compare the tomographic data to the mercury intrusion data of the same samples (Fig. 11).

The volumic fractions of pores larger than $15 \mu \mathrm{m}$ measured with both techniques are presented in Table 3 .
TABLE 2

Volumic fraction of pores and minerals as deduced from global
measurements (Durand, 1996) and measurements
from grey level histograms

\begin{tabular}{c|c|c|c|c|c|c}
\cline { 2 - 7 } & \multicolumn{2}{c|}{ Sample CAR1 } & \multicolumn{2}{c|}{ Sample CAR3 } & \multicolumn{2}{c}{ Sample CAR4 } \\
\hline $\begin{array}{c}\text { Volume } \\
\text { fraction }\end{array}$ & $\begin{array}{c}\text { Global } \\
(\% \mathrm{vol})\end{array}$ & $\begin{array}{c}\text { From hist. } \\
(\% \mathrm{vol})\end{array}$ & $\begin{array}{c}\text { Global } \\
(\% \mathrm{vol})\end{array}$ & $\begin{array}{c}\text { From hist. } \\
(\% \mathrm{vol})\end{array}$ & $\begin{array}{c}\text { Global } \\
(\% \mathrm{vol})\end{array}$ & $\begin{array}{c}\text { From hist. } \\
(\% \mathrm{vol})\end{array}$ \\
\hline $\begin{array}{c}\text { Pores } \\
>15 \mu \mathrm{m}\end{array}$ & 16 & 13 & 17 & 11 & 22.6 & 10 \\
\hline $\begin{array}{c}\text { Pores } \\
<15 \mu \mathrm{m}\end{array}$ & 16 & 16 & 17 & 9 & 22.6 & 16 \\
\hline Clays & 12 & 16 & 2.5 & 9 & 5 & 16 \\
\hline $\begin{array}{c}\text { Total: } \\
\text { pores } \\
+ \text { clays }\end{array}$ & 28 & 29 & 19.5 & 20 & 27.6 & 26 \\
\hline Quartz & 71 & 71 & 77 & 80 & 62 & 64 \\
\hline Feldspars & - & & 2.6 & - & 10.5 & 10 \\
\hline
\end{tabular}

TABLE 3

Comparison between microscanner and $\mathrm{Hg}$ porosimetry data

\begin{tabular}{c|c|c|c|c}
\hline Sample & $\begin{array}{c}P: \text { total } \\
\text { pore volume } \\
(\text { from Table I) }\end{array}$ & $\begin{array}{c}P_{15}: \text { measured } \\
\text { pore volume } \\
\text { Pores }>15 \mu \mathrm{m}\end{array}$ & $\begin{array}{c}\text { Measured } \\
\text { pore fraction } \\
P_{15} / P\end{array}$ & $\begin{array}{c}\text { Hg intrusion } \\
\text { pore fraction } \\
\text { Pores }>15 \mu \mathrm{m}\end{array}$ \\
\hline CAR1 & $23 \%$ & $10 \%$ & $40 \%$ & $40 \%$ \\
\hline CAR3 & $17 \%$ & $11 \%$ & $64 \%$ & $50 \%$ \\
\hline CAR1 & $16 \%$ & $13 \%$ & $80 \%$ & $30 \%$ \\
\hline
\end{tabular}

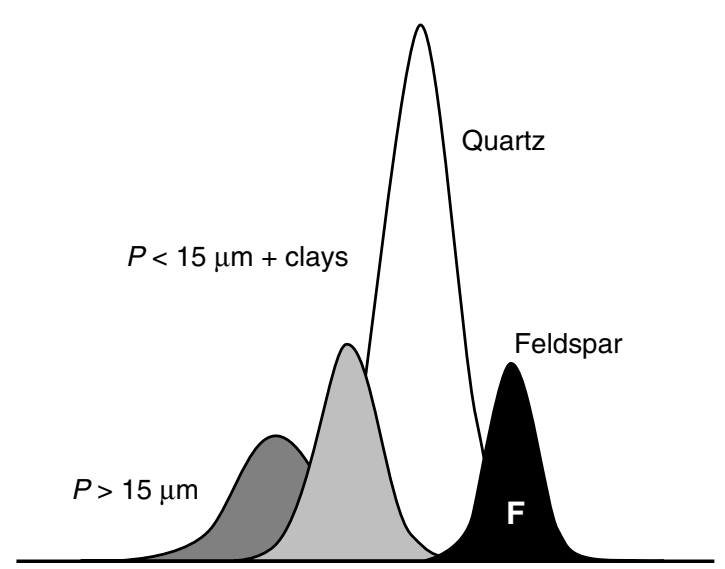

Figure 10

Grey level histogram of sample CAR4.

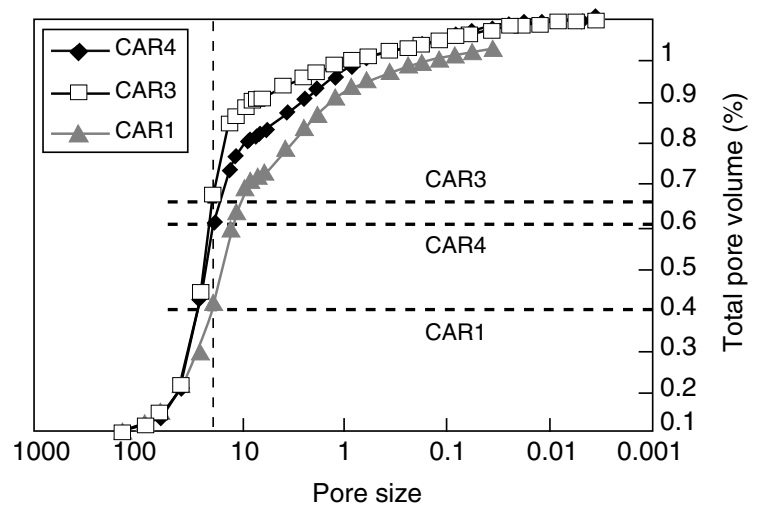

Figure 11

Mercury intrusion data. 
We find a good agreement for samples CAR4 and CAR3, but no agreement in the case of sample CAR1 for which microtomography detects $80 \%$ of pores larger than $15 \mu \mathrm{m}$ while mercury porosimetry only detects $30 \%$ of pores larger than $15 \mu \mathrm{m}$. This apparent contradiction is due to the fact that pore definition is not the same in both cases. Mercury porosimetry measures the volume accessible through pore throats larger than $15 \mu \mathrm{m}$, while microscanner measures the whole volume of pores larger than $15 \mu \mathrm{m}$ (chambers and throats).

Imaging the pore networks help us to understand this apparent contradiction. Pore network representations show that samples CAR4 (Fig. 12) and CAR3 (not presented) have a pore structure which can be assimilated to bundles of interconnected capillary tubes (corresponding to the model used in mercury porosimetry calculations) whilst CAR1 has much larger macropores with very few connections visible at this scale. This topology difference was not suggested neither by 2D SEM images (Fig. 9a, 9b, 9c) neither by $\mathrm{Hg}$ intrusion measurements (Fig. 11).
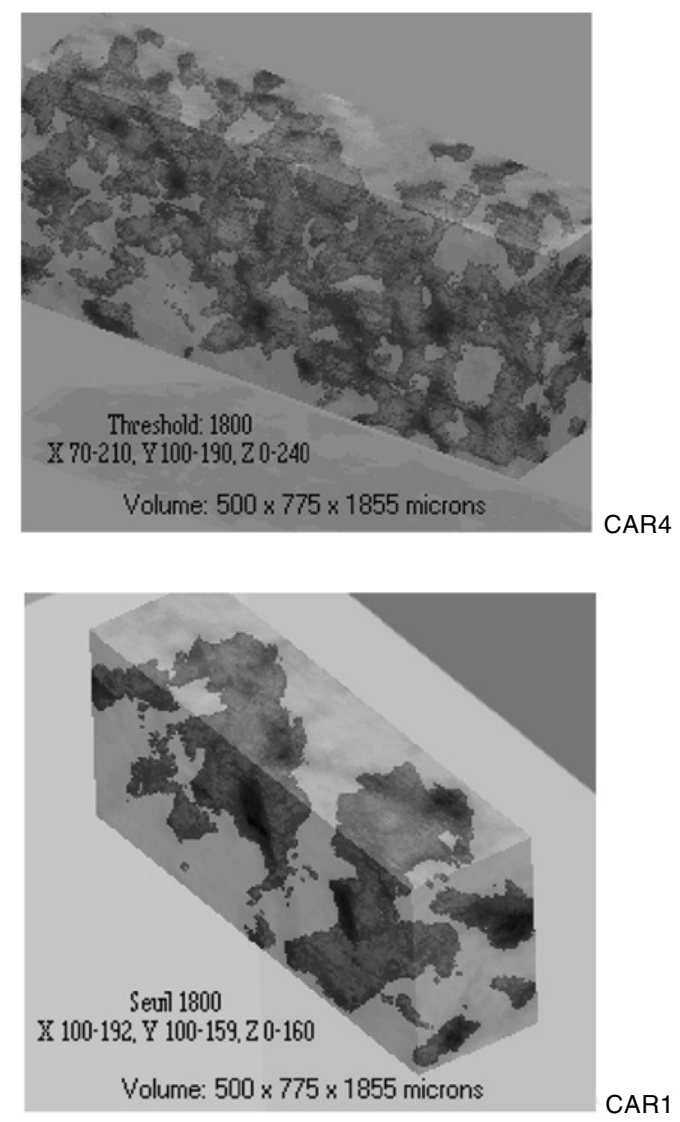

Figure 12

Reconstructed pore volume on two rock samples (CAR4 and CAR1). The threshold used for segmentation is the intersection between the first and the second peak of the grey level histogram. The isolated pore networks are represented in a fixed dimension volume
From these data, the low permeability of sample CAR1 is clearly correlated to the very few connections between the pores and to the large pore to throat ratio.

If we look at the short range connections by representing the pore network on a few contiguous slices (Fig. 13) we can notice differences between sample CAR3 and CAR4. The connections are numerous in the case of CAR4 even on an $80 \mu \mathrm{m}$ thick block (Fig. 13b) whilst in the case of CAR3, they are not visible on a $160 \mu \mathrm{m}$ thick slice (Fig. 13c and 13d) and begin to be seen only for a $320 \mu \mathrm{m}$ thick slice (Fig. 13e and 13f). Here, the difference in permeability is correlated with the range of interconnections of the pores.

Comparison of 3D porous networks in these media of different permeability gives important information about the structure of the network, particularly concerning the topology, the throat to chamber ratios, and the connectivity of pores. Sample CAR1 presents very large pores confined by very small throats (below the resolution limit). From this pore structure, oil wettability of the rock CAR1 when compared to CAR3 may be explained in two ways:

- these fine throats may impose a high capillary pressure for oil to penetrate into macropores and this pressure may result in breaking of water films along the pore walls. Oil wettability of CAR1 would result in this case from a geometric effect;

- capillary pressure is not sufficient to break water films in either pore structure and the oil wet character of CAR1 would result from a mineralogical effect (presence of clays).

The information currently available does not allow us to fully eliminate one of these possibilities.

As a conclusion of this part of the study, microtomography gives informations about the 3D structure of the pore or mineralogical network. We found a good qualitative agreement between permeabilities and the $3 \mathrm{D}$ pore structure directly available from microtomography measurement. We found differences in the number of interconnections, their range and the pore/throat ratio. Mineral identification and distribution have been obtained from attenuation values and confirmed by tandem SEM observations. We encountered some difficulties with kaolinite due to its size near to the resolution and its attenuation very close to that of quartz. In favourable cases, kaolinite rich areas were reliably reconstructed from tomographic data (Fig. $7 \mathrm{~g}$ ). The opportunity to detect them will depend on the way they are aggregated. Kaolinite aggregates can be mistaken for quartz or porosity, depending on their sizes and separation.

An improvement in resolution would be useful to describe pore connectivity in low permeability media. A resolution of $2 \mu \mathrm{m}$ has been reached on projections (Ferreira De Paiva, 1996) and this can be considered as the actual resolution limit for this microtomograph. Nevertheless, the acquisition mode and number of projections imply a compromise between the resolution and the sample size. To reach $2 \mu \mathrm{m}$ resolution implies working on very small volumes from which extrapolation to higher scales is difficult. 

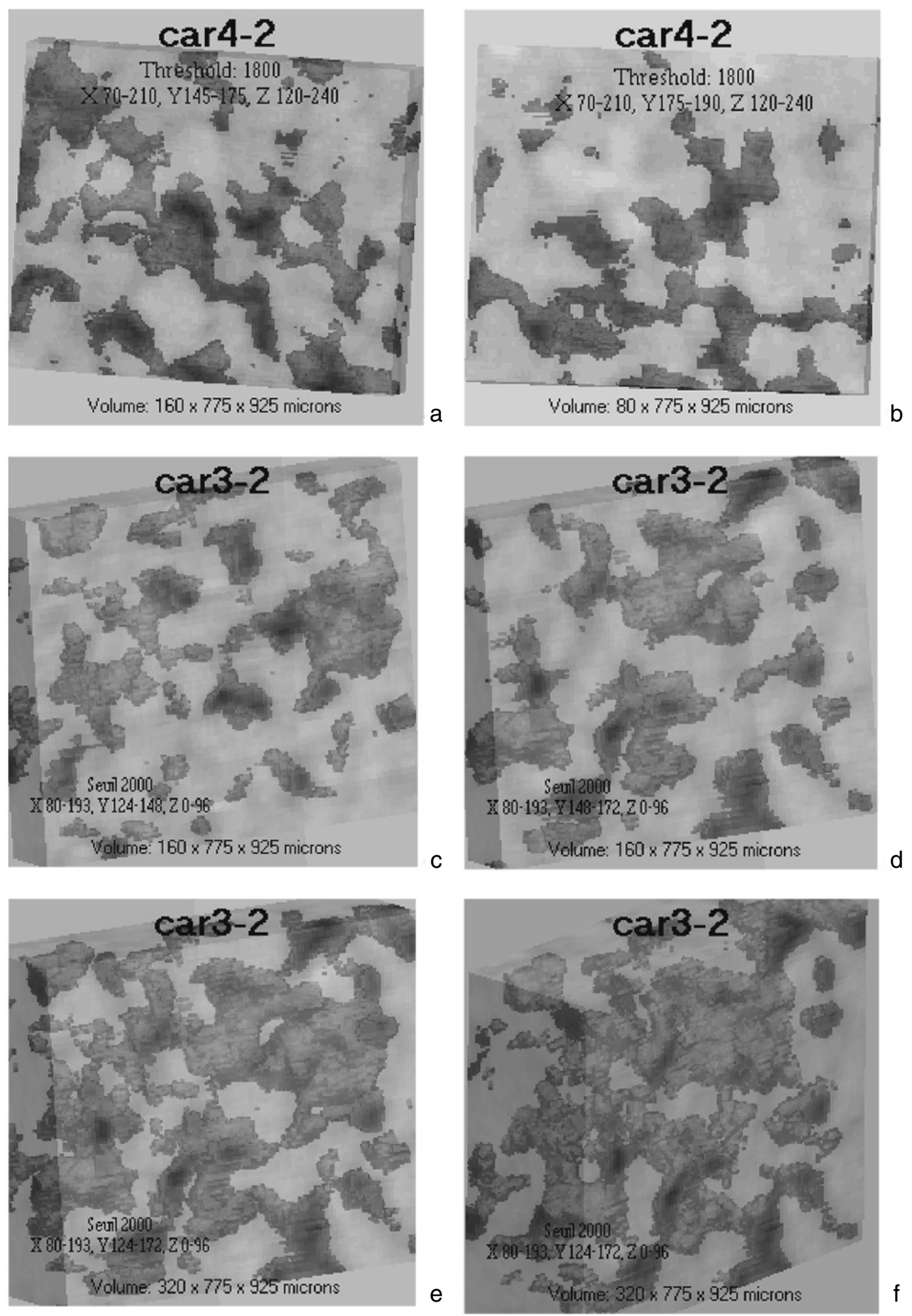

Figure 13

Pore network of samples CAR3 and CAR4. Representation of the pore network on a few contiguous slices (short range connections). a, b: 160 and $80 \mu \mathrm{m}$ thick block of sample CAR4 showing many connections; c, d: $160 \mu \mathrm{m}$ thick block of sample CAR3 showing few connections; e, f: $320 \mu \mathrm{m}$ thick block of sample CAR3 showing connection between pores. 
Samples studied here were 1 to $2 \mathrm{~mm}$ in diameter and $3 \mathrm{~mm}$ long. This appeared to be a good compromise between the required resolution and the representativity of the samples. In the case of heterogeneous specimens, a number of representative samples per rock should be studied. This condition is not however limiting when laboratory equipment is available.

Tridimensional data available from reconstructions are a good departure point for many theoretical studies such as boundary conditions in fluid flow modeling, identifying departure from the capillary tube geometry assumed in $\mathrm{Hg}$ porosimetry, reconciliating 2D information from SEM images and global measurements like porosimetry, understanding the effects of wettability on capillary pressure curves or interpreting saturation measurements.

\subsection{Glass Fiber Reinforced Thermoplastics}

Short fibre reinforced thermoplastics are commercially available and, because of their low cost, are finding widespread acceptance for many applications. In order to improve their mechanical properties, the glass fibres should be oriented in the direction of the applied stress. One of the means to reach a given orientation state is to use an extrusion process. Many extrusions parameters such as die geometry (Fig. 14), or use of a lubricant, may have an influence on the fibres angular distribution. It is therefore of critical importance to be able to control the 3D orientation of the fibres in such flow processes.

The microscanner enables a methodology to be developed for the study of fibre orientation in extruded composites. Resolution of our apparatus is sufficient to visualise individual fibres. An example of $3 \mathrm{D}$ reconstruction is given in Figure 15. Figures 15a and $15 \mathrm{~b}$ concern $20 \mu \mathrm{m}$ diameter glass fibres while Figures $15 \mathrm{c}$ and $15 \mathrm{~d}$ concern $10 \mu \mathrm{m}$ diameter glass fibres. The resolution enables in both cases to resolve the fibres and thus microtomography appears as a method of choice to characterise fibre orientation.

A preliminary study was undertaken to understand the influence of extrusion die geometry and the influence of extrusion parameters on fibre orientation. Particularly interesting was the determination of the orientation of fibres in hollow tubes extruded with a diverging die, designed in order to achieve a circonferential orientation of fibres within the tube (Fig. 14).

The materials studied were composites made of a polyethylene matrix reinforced with $15 \%$ volume glass fibres of $10 \mu \mathrm{m}$ diameter and about $400 \mu \mathrm{m}$ long. Composites were in the form of extruded hollow tubes, $40 \mathrm{~mm}$ in diameter and 1 to $3 \mathrm{~mm}$ thick.

Radiographic acquisitions were made using a $3 \mu \mathrm{m}$ thick copper target, $30 \mathrm{kV}$ accelerating voltage, and a current intensity of $300 \mathrm{nA}$. A carbon filter, $1000 \mu \mathrm{m}$ thick was used

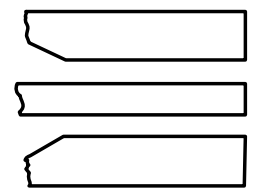

Classical die geometry

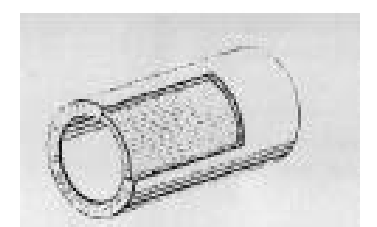

Orientation parallel to the axis

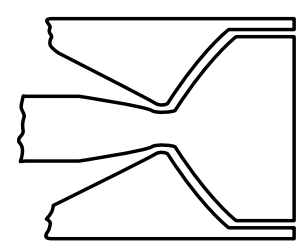

Diverging die geometry

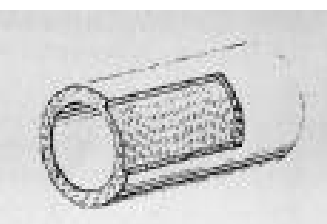

Circonferential orientation
Figure 14

Expected influence of the die geometry on the fibres orientation.

in order to reduce beam hardening effects. The reconstructed sections show that in all cases fibres are mainly oriented in the orthoradial plane (Fig. 16).

Fibres orientations were thus measured in this plane (Fig. 16). For each section, we calculated the orientation tensor component defined as:

where $n$ is the number of measured fibres and $\alpha_{i}$, the angle between each fibre and the $z$ axis. This parameter, defined in a previous work by G. Ausias (Ausias, 1994) enables a differentiation between an orientation parallel to the axis of the tube, and a circonferential orientation.

$a_{\theta \theta}=0$ when the orientation is parallel to the tube axis

$a_{\theta \theta}=1$ when orientation is circonferential.

We took advantage of the fact that we dispose for each sample of a set of contiguous sections to precisely compare the orientation of fibres across the thickness of hollow tubes prepared in different extrusion conditions (different die geometry, different pulling velocity and addition of a lubricant).

Reconstructed sections show that orientation is different close to the walls of the tube and in the central part of the tube, and is sometimes different on the two walls (parts in contact with the walls of the die).

- In the die with the classical parallel geometry (Fig. 17) fibres orient mostly parallel to the axis of the tube near the walls and are more disordered in the central part of the tube. A higher pulling velocity at the exit of the die makes the central fibres orient parallel to the axis of the tube. 

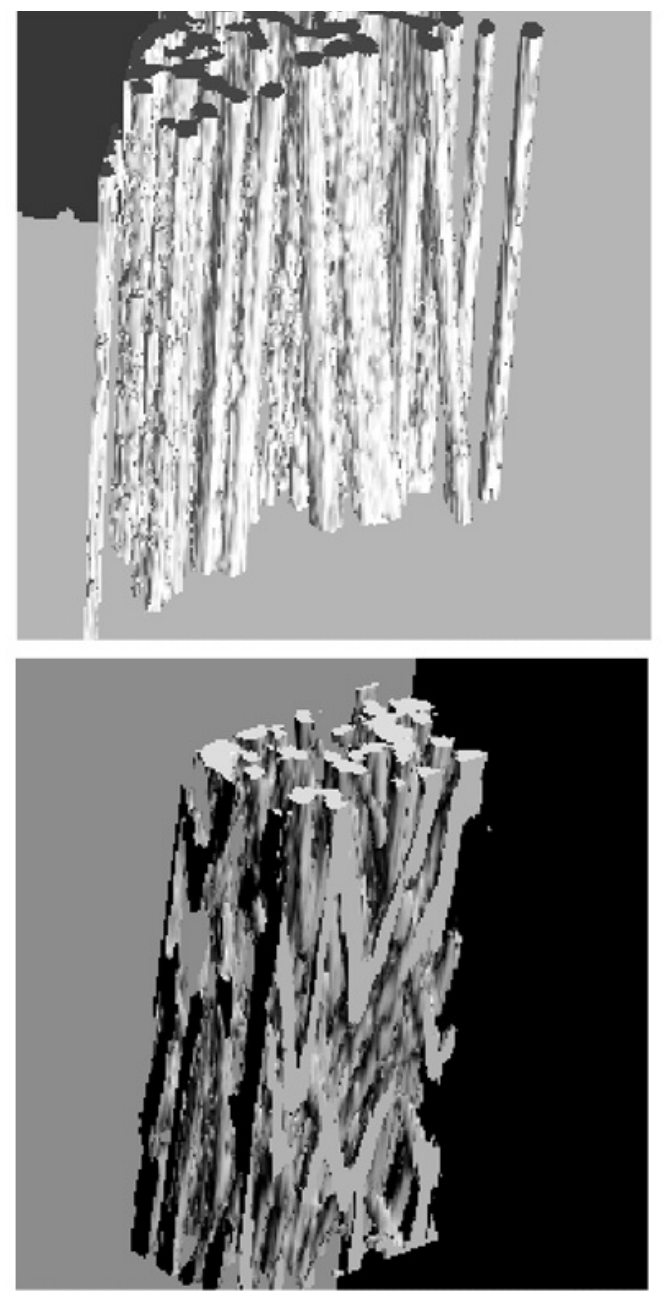

a

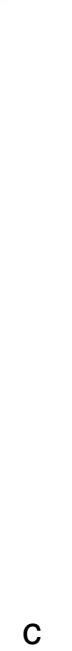

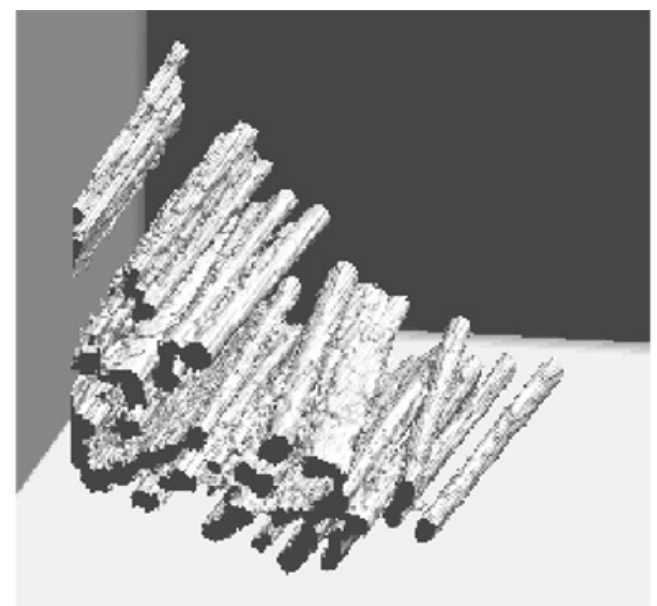

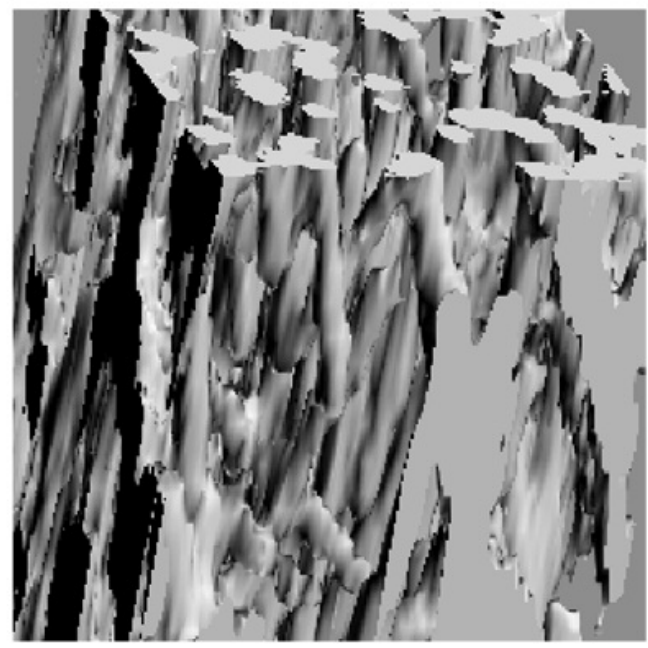

b

d

Figure 15

Visualisation of glass fibres in composites. a, b: $20 \mu \mathrm{m}$ diameter fibres; $\mathrm{c}, \mathrm{d}:: 10 \mu \mathrm{m}$ diameter fibres.

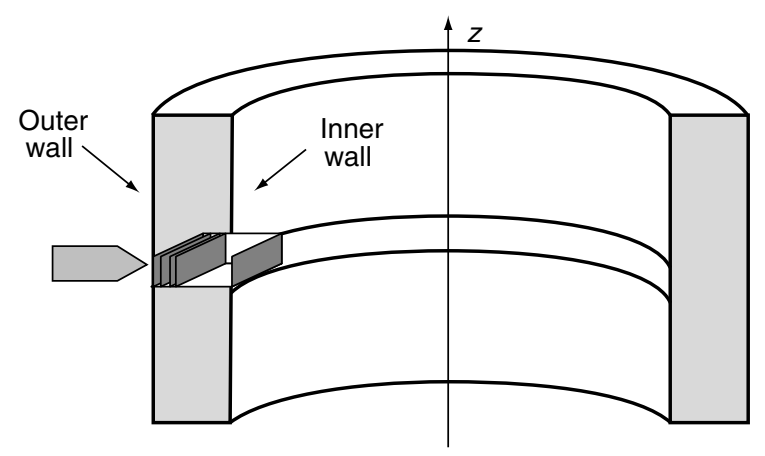

Figure 16

Arrangement of serial orthoradial sections in the thickness of the tube.
- In the diverging die (Fig. 18), fibres no longer show longitudinal orientation near the walls but do not have exactly the ideal circonferential orientation. Using no lubricant, fibres orient parallel to the tube axis near the walls and at random (but still in the orthoradial plane) in the central part of the tube. When a lubricant is introduced along the walls of the die, the orientation of fibres become preferentially circonferential, particularly in the central part of the tube.

Although preliminary, these results reveal interesting information for improving the design of the die geometry and adjusting extrusion parameters when a specific orientation of the fibres is required. One of the main advantages of the microscanner is to produce up to 256 sections with a high positioning precision across the thickness of the tube with interesting consequences. First, the representation of data in three orthogonal planes directly indicates the preferential orientation of the fibres, and if fibres are preferentially oriented 


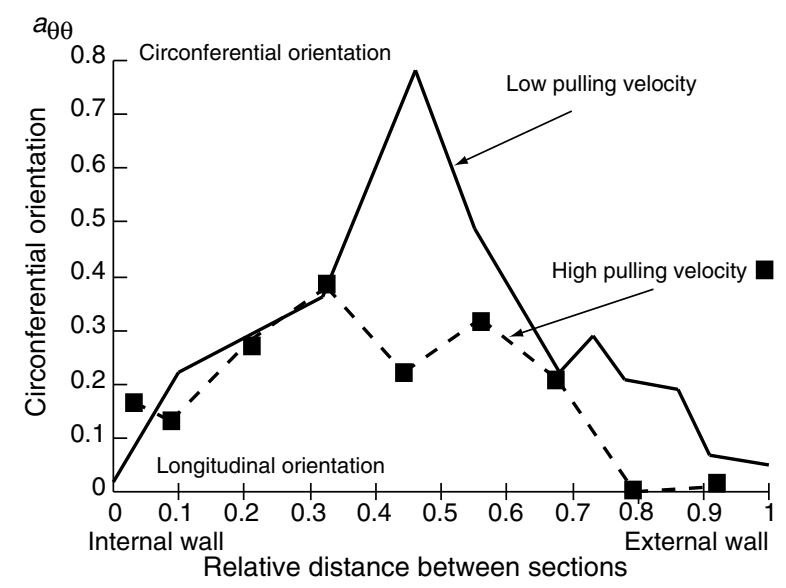

Figure 17

Orientation profile of fibres across the thickness of the tube. Parallel die geometry. Use of different pulling velocity at the exit of the die. $a_{\theta \theta}$ is measured in successive orthoradial planes; $a_{\theta \theta}=0$ the orientation is parallel to the tube axis, $a_{\theta \theta}=1$ the orientation is circonferential.

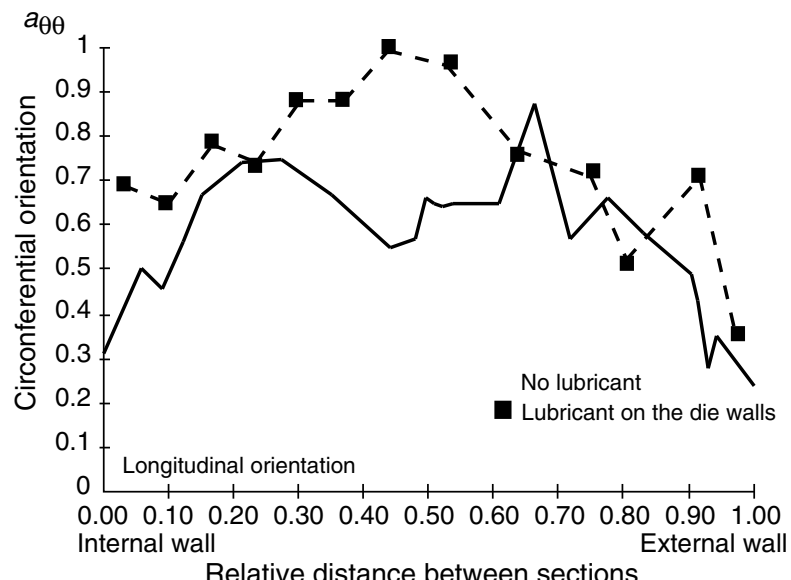

Figure 18

Orientation profile of fibres across the thickness of the tube. Divergent die geometry. $a_{\theta \theta}$ is measured in successive orthoradial planes.

in a plane (here fibres were preferentially oriented in the orthoradial plane) angular measurements can be done in this plane. Secondly measurements made on joined sections can be regarded as repeatability measurements. Finally the orientation profiles across the section of the tube can be compared to the calculated velocity profiles for each die geometry and give an experimental validation of theoretical models.

\section{CONCLUSIONS}

This laboratory microscanner allows in its standard use a resolution of about $10 \mu \mathrm{m}$ which already enables the study of some reservoir rocks, giving directly 3D connectivity or topology information on the pore network. The quality of the reconstructions have been proved by comparing SEM images of the sample with the reconstructed surface of the sample. The qualitative information from the $3 \mathrm{D}$ reconstructions of pore networks (shape, topology, pore volume, connections) provides a unique, visual support to reconciliate 2D microscopy measurements and global data like porosimetry or permeability measurements. The semi-quantitative data (porosity, or phase concentration measurements) can be correlated with global measurements, a good agreement ensuring the good representativity of the microscanner sampling. This technique also reveals interesting in the study of $3 \mathrm{D}$ orientation of fibres in composite samples allowing for example optimisation of the extrusion processes parameters. The limits of the technique are also clear from these examples. As with many microscopy techniques, resolution is sometimes insufficient (illustrated on kaolinite) leading to the averaging of the information on heterogeneities at a scale below the resolution, and the size of samples (limited to a few millimetres ) is often critical. Nevertheless the data issued from this technique must be seen as an help for many theoretical studies or as a visual support for interpretation of global measurements like $\mathrm{Hg}$ porosimetry or NMR relaxation measurements.

\section{ACKNOWLEDGMENTS}

The authors are grateful to M. Graf, for the modifications of the apparatus, they would like to thank Mrs Martins for her participation in the experimental study, Mrs Durand and MM. Jarrin, Dalmaso and Cartalos for furnishing interesting examples of industrial problems.

\section{REFERENCES}

Ausias, G., Agassant, J.F. and Vincent, M. (1994) Flow and fiber orientation calculations in reinforced thermoplastic extruded tubes. Inter. Polymer Processing IX, 51-59, Munich.

Cazaux, J., Thomas, X. and Mouse, D. (1989) Microscopie et microtomographie X. J. Microsc. électronique, 14, 263-276.

Cheng, P.C., Shinozakid, M., Lin, T.H., Newburry, S.P., Sridhar, R., Tarng, W., Chen, M.T and Chen, L.H. (1992) X-Ray Microscopy III. Edited by A. Michette et al. Springer, Berlin.

Coles, M.E., Spanne, P., Muegge, E.L. and Jones, K.W. (1994) Computed Microtomography of Reservoir Core Samples. SCA Paper No. 9401, presented at 1994 Intern. Symp. of SCA, Stavenger, 9-16.

Coles, M.E., Hazlett, R.D., Spanne, P., Soll, W.E., Muegge, E.L. and Jones, K.W. (1996) Pore Level Imaging of Fluid Transport Using Synchrotron X-ray Microtomography. SCA Paper No. 9628, presented at 1996 Intern. Symp. of SCA, Montpellier, France. 
Cuiec, L. (1991) Evaluation of Reservoir Wettability and its Effects on Oil Recovery. Interfacial Phenomena in Petroleum Recovery, Ed. N.R. Morrow, Marcel Dekker Inc, N.Y.

Durand, C. and Rosenberg, E. (1996) Fluid Distribution in Kaolinite or Illite-Bearing Cores. Cryo-SEM Observations Versus Bulk Measurements. SCA Paper No. 9627, presented at 1996 Intern. Symp. of SCA, Montpellier, France.

Fassi Fihri, O., Robin, M. and Rosenberg E. (1992) Étude de la mouillabilité des roches-réservoirs à l'échelle du pore par cryomicroscopie à balayage. Revue de l'IFP, Éditions Technip, 37, 5, 685-701.
Ferreira de Paiva, R. (1995) Developpement d'un microtomographe $\mathrm{X}$ et application à la caractérisation des roches-réservoirs. Thesis Université of Paris VI, France.

Ferreira de Paiva, R., Bisiaux, M., Lynch, J. and Rosenberg, E. (1996) High Resolution X-Ray Tomography in an Electron Microprobe. Rev. Sci. Instrum., 67, 6.

Sasov, A.Y. (1987) Microtomography. J. Microscopy, 147, 169178 and $179-192$. 
